\title{
Decay correlations in the seconds range with laser-ionized, mass-separated beams
}

\author{
D. Pauwels ${ }^{\mathrm{a}, *}$, O. Ivanov ${ }^{\mathrm{a}}$, J. Büscher ${ }^{\text {a }}$, T.E. Cocolios $^{\text {a }}$, J. Gentens ${ }^{\mathrm{a}}$, M. Huyse ${ }^{\mathrm{a}}$, A. Korgul ${ }^{\mathrm{b}}$, \\ Yu. Kudryavtsev ${ }^{\text {a }}$, R. Raabe ${ }^{\text {a }}$, M. Sawicka ${ }^{\text {a }}$, I. Stefanescu ${ }^{a}$, J. Van de Walle ${ }^{\text {a }}$, \\ P. Van den Bergh ${ }^{\mathrm{a}}$, P. Van Duppen ${ }^{\mathrm{a}}$ \\ ${ }^{\text {a } I n s t i t u u t ~ v o o r ~ K e r n-~ e n ~ S t r a l i n g s f y s i c a, ~ K . ~ U . ~ L e u v e n, ~ C e l e s t i j n e n l a a n ~ 200 D, ~ B-3001 ~ L e u v e n, ~ B e l g i u m ~}$ \\ ${ }^{\mathrm{b}}$ Institute of Experimental Physics, Warsaw University, ul.Hoża 69, 00-681 Warszawa, Poland
}

Available online 6 June 2008

\begin{abstract}
The upgraded LISOL $\beta$-decay detection setup, which was used for the measurement of the mass $A=67 \mathrm{Fe}-\mathrm{Co}-\mathrm{Ni}$ decay chain, is presented. A new decay correlation technique has been developed in order to characterize an isomeric $492 \mathrm{keV}$ transition, observed in this decay chain. Correlating single $\beta$ and $\gamma$ events and $\beta$-coincident $\gamma$ events with each other in the seconds range, allowed for the placement of this isomeric transition in ${ }^{67} \mathrm{Co}$. A new half-life for the ${ }^{67} \mathrm{Co}$ ground state was determined. The technique and its possibilities and limitations are discussed.
\end{abstract}

(c) 2008 Elsevier B.V. All rights reserved.

PACS: $07.05 . \mathrm{Kf} ; 23.35 .+\mathrm{g} ; 23.40 .-\mathrm{s} ; 27.50 .+\mathrm{e}$

Keywords: Correlations; $\beta$-decay; ISOL

\section{Introduction}

Implantation-decay correlations are a very powerful technique at in-flight separators to reach out far in the region of unstable nuclei, see e.g. [1-3]. With the advent of post-accelerated ISOL beams this technique has also successfully been used for the observation of rare charged-particle decay channels, see [4]. At low-energy ISOL systems, however, the acceleration voltage (typically $60 \mathrm{kV}$ ) is not high enough to give an unambiguous implantation signal and often the beam of interest is overwhelmed by unwanted radioactive or stable contaminants. Instead, the decays are classically studied on the basis of coincidences of a particle (beta, neutron, proton, alpha) with a $\gamma$-event, two $\gamma$-events or even higher multiplicity combinations, where the coincidence time window ranges in the order of nanoseconds up to at maximum milliseconds.

\footnotetext{
${ }^{*}$ Corresponding author.

E-mail address: Dieter.Pauwels@fys.kuleuven.be (D. Pauwels).
}

Longer coincidence time windows are restricted by the amount of random coincidence events hampering the true correlations and thus causing a loss in sensitivity.

For decay studies at ISOL facilities, this puts a severe restriction to investigate the properties of supra-milliseconds isomeric states. The longer the half-life is, the larger the coincidence window has to be chosen and the more the coincident events are getting drowned in the random counts. However, with the decay correlation technique that will be presented here, combined with weak but pure sources and the flexibility of element selectivity using lasers, it becomes possible to accurately subtract the random activity for decays up into the seconds range. In that way, the exponential decay of the physically correlated events are made random-free. As today's nuclear structure study is mainly aimed at regions far from the line of stability, where the typical $\beta$-decay half-lives are in the order of seconds or even smaller, the correlation technique can extensively be exploited to investigate their respective ground and isomeric states. 
This technique has been used for the first time on data recorded during the ${ }^{67} \mathrm{Fe} \beta$-decay experiment at the Leuven Isotope Separator On Line (LISOL) facility in Louvain-LaNeuve, Belgium, [5,6]. The segmented detection system, digitally read-out, in combination with selective laser-ionization and on-line mass separation has created the possibility at low-energy ISOL separators for decay correlations in the seconds range. Performing a sequence of experiments with the lasers selectively tuned to $\mathrm{Fe}, \mathrm{Co}$ and $\mathrm{Ni}$, respectively, and making use of the new technique of mother, daughter and even granddaughter correlations in the seconds time domain, allowed us to disentangle this complicated decay chain and identify an isomer with a half-life in the order of $0.5 \mathrm{~s}$ in ${ }^{67} \mathrm{Co}$, while its ground state has a half-life of 329(28) ms. The correlation technique allowed us to deduce the ${ }^{67} \mathrm{Co}$ level scheme, the half-lives involved and their $\beta$ branching ratios.

\section{The detection setup}

The current LISOL detection setup, shown in Fig. 1, is based on the setup described in [7]. The mass-separated ions are implanted in the implantation tape, where their subsequent $\beta$-decay is detected by the surrounding $\beta$ - and $\gamma$-detectors. Typically one runs in cycles of a specific implantation time period followed by a specific decay time period when the cyclotron beam is closed. The implantation and decay times are chosen according to the half-life of the investigated nucleus. To suppress the effect of long-lived contaminants the implantation tape is moved after a certain number of such implantation-decay cycles.

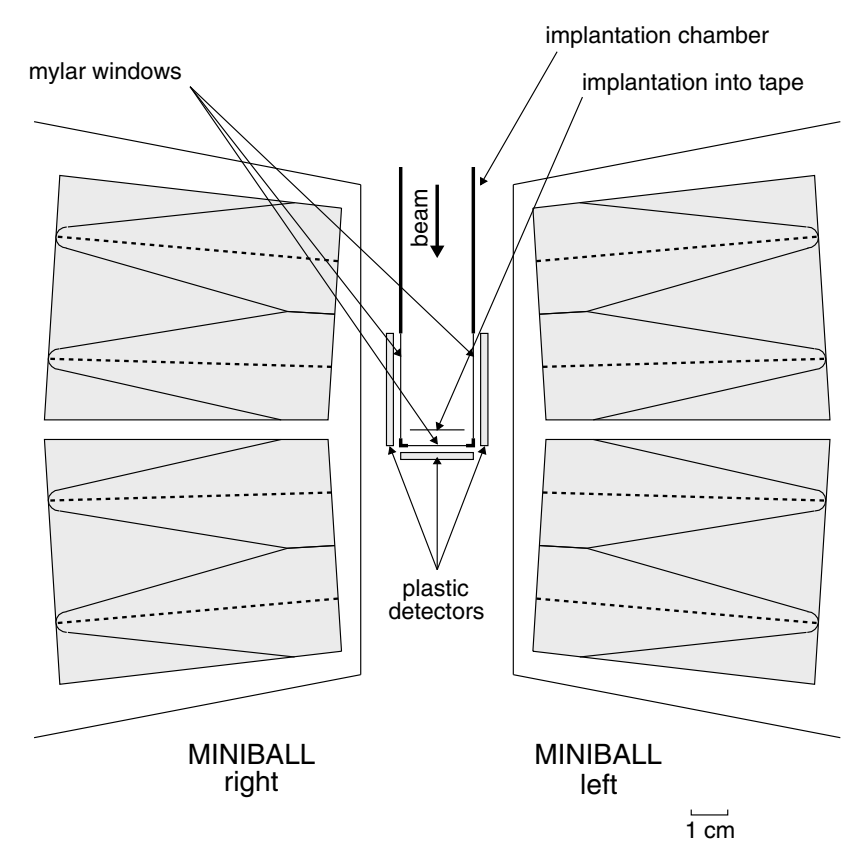

Fig. 1. A schematic drawing of the top view of the detection setup. The two MINIBALL clusters and three plastic detectors are indicated. Electric segmentation is indicated by the dotted lines.
The detection setup has been upgraded and some important differences are discussed below.

Firstly the two coaxial Ge $\gamma$-ray detectors with $70 \%$ and $75 \%$ relative efficiency are replaced by two MINIBALL clusters [8], each composed of three HPGe crystals. One crystal has a relative efficiency of $55 \%$ and is electrically sixfold segmented as indicated by the dotted lines in Fig. 1. The solid angle that is covered by one MINIBALL crystal from the implantation spot is on the order of $9 \%$ of $4 \pi$. For the total of six crystals this is very similar to the total solid angle covered by the two coaxial Ge detectors (in the order of $60 \%$ of $4 \pi$ ). The reason for the similar total covered solid angle is mainly the larger distance of the MINIBALL Ge crystals from the implantation spot and, to a smaller degree, the geometry. Despite the fact that there is no gain in total solid angle, there is a gain in photo-peak efficiency, because of the reduction of true summing. The probability of two coincident $\gamma$-rays entering the same crystal is $0.8 \%$ for the MINIBALL setup, while it is $9 \%$ for the coaxial detector setup. With this MINIBALL setup the photopeak efficiency of a multiplicity $1 \gamma$-ray of $1332 \mathrm{keV}$ is $5.8 \%$.

A second difference is the new electronics and dataacquisition system. To read-out the $42 \mathrm{Ge}$ signals ${ }^{1}$ digital electronics are used. The MINIBALL preamplifier signals are digitized and processed by XIA-DGF4C modules [9] on an event-by-event basis. Each module has four preamplifier signal inputs and an internal $40 \mathrm{MHz}$ clock. The modules are initialized and synchronized by the IGOR software package [10]. Our data-acquisition system is running in the format of storing energy and time information for each event.

The $\beta$-particles are detected with an efficiency of $50 \%$ by three thin plastic scintillators that cover $68 \%$ of $4 \pi$. Lower energy particles in the $\beta$-distribution are only partially registered causing an overall intrinsic efficiency that stays constant at about $73 \%$ for decays with a $\beta$-endpoint energy larger than $4 \mathrm{MeV}$. The plastic detectors serve as a $\beta$ trigger only and the energy information is not registered, see [7] for more details.

\section{The correlation technique}

With the LISOL detection setup, there are three possible event types that can be correlated with each other: single $\gamma$ events, single $\beta$-events and $\beta$-coincident $\gamma$-events inside a time window of $50 \mathrm{~ns}<t_{\gamma}-t_{\beta}<400 \mathrm{~ns}$ (the $\beta \gamma$-events). Three steps are required for building a random-free correlation spectrum.

In the first step, the exact trigger event with the $\gamma$-energy gate, in case a $\gamma$-signal is involved and the correlation event type have to be specified. All the trigger events that are pres-

\footnotetext{
${ }^{1}$ Each crystal has seven signals (6 segment signals and 1 core signal, which collects all the energy deposited in the total crystal) and in total there are six crystals. However there are also MINIBALL clusters available with an additional twofold segmentation in the longitudinal direction. In total this means 13 signals per crystal.
} 
ent in the data are scanned. Every time a valid trigger event is found, correlated histograms are built in equal time slices before and/or after the trigger event, within a correlation time window of typically two to four times the correlation half-life, but outside the prompt trigger event window (typically $0.5 \mu \mathrm{s})$. The result is a set of histograms with the desired correlations, but still drowned in random correlations.

The random correlations can accurately be approximated though in the second step, even with the specific implantation-decay cycles at LISOL, where isobaric contaminants with different half-lives have different growingin and decay structures. Every trigger event occurred at a specific time inside the implantation-decay cycle. The time windows within the cycle of the corresponding correlated histograms are therefore also determined. Since the implantation-decay structure is identical for each cycle, the randomly correlated activity in the correlated histograms can accurately be approximated by the events within these specific time windows for the total statistics of all cycles, normalized to the number of cycles.

In the final third step, the histograms containing the approximated randomly correlated events are subtracted from the correlated histograms. The results are the random-free correlated histograms, which show the evolution of the physically correlated events as a function of time with respect to the trigger event.

Different kind of correlation techniques have become a common issue in today's nuclear physics. The presented technique differs with other known techniques in the fact that the trigger event is not an implantation signal or a characteristic charged-particle signal like alphas, $\beta$-delayed nucleons and direct nucleons, but rather a less specific signal like a single $\gamma$ - or $\beta$-signal or a $\beta \gamma$-signal. Because the trigger is in this case less characteristic, the accurate knowledge of the random correlations becomes mandatory.

\section{Limitations}

Obviously the presented technique has its limitations. Before going into more details, definitions are needed for the different types of correlation and trigger events. For convenience this will be done making use of Fig. 2. Suppose all events within the $\gamma_{1}$ photo-peak energy window are specified as trigger events and its correlation with the

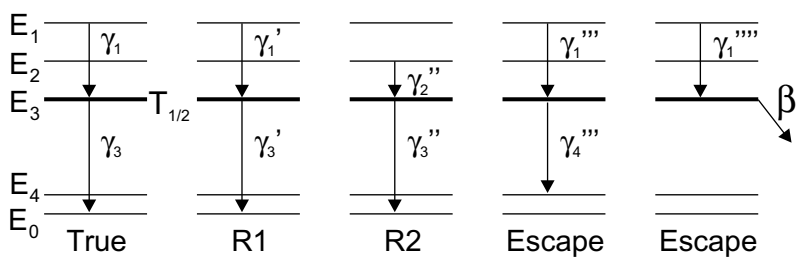

Fig. 2. An example of five hypothetical consecutive $\gamma$-ray decay cascades is shown. An isomeric state at energy $E_{3}$ de-excites with a half-life $T_{1 / 2}$. In the text the situation is discussed for the correlation time window that is opened by $\gamma_{1}$. $\gamma_{3}$-transition is investigated. In the following the situation is discussed for the correlation time window $\Delta t$ that is opened by the $\gamma_{1}$-trigger observed at time $t_{0}$.

- The true correlation event (indicated as "True" in the figure) is the detection within this correlation time window of $\gamma_{3}$. All other correlation events are random correlations.

- The first type of random correlations, indicated as " $R_{1}$ " and " $R_{2}$ " in the figure, is the detection within the correlation time window of $\gamma_{3}^{\prime}$, respectively, $\gamma_{3}^{\prime \prime}$ coming from another decaying nucleus of the same type fed through the preceding $\gamma_{1}^{\prime}$, respectively, $\gamma_{2}^{\prime \prime}$. In the " $R_{2}$ " case, it is more generally required that the randomly correlated event is an event of the same type as a truly correlated event, but belonging to a decay cascade that does not contain the transition of the type of the trigger event.

- The second type of a random correlation, labeled in the text by the subscript "bg", but not shown in Fig. 2, is the detection within the correlation time window of a background event inside the correlation energy window.

So far, the different types of correlated events are discussed, but also three different types of trigger events exist.

- A true trigger event is a trigger event that is followed by the investigated correlated event, irrespective of the fact that the correlated event gets detected or not. In Fig. 2 only $\gamma_{1}$ and $\gamma_{1}^{\prime}$ are true trigger events.

- The first type of a faulty trigger event is the detection of transitions, like $\gamma_{1}^{\prime \prime \prime}$ and $\gamma_{1}^{\prime \prime \prime}$ in the figure, which are not followed by the investigated correlation event. One can speak of an escape out of the $\left(\gamma_{1}-\gamma_{3}\right)$ correlation scheme.

- The second type of a faulty trigger event (not shown in Fig. 2) is a background event inside the trigger photopeak energy window.

The total number of true correlations $N_{\text {true }}^{\mathrm{c}}$ can be expressed by the equation:

$N_{\text {true }}^{\mathrm{c}}=N_{\text {true }}^{\mathrm{tr}} I_{\mathrm{b}} \varepsilon_{\mathrm{c}}\left(1-\mathrm{e}^{-\lambda \Delta t}\right)$,

where $N_{\text {true }}^{\text {tr }}$ is the number of true trigger events, $I_{\mathrm{b}}$ is the branching ratio, $\varepsilon_{\mathrm{c}}$ the detector efficiency for the correlation events, $\lambda=\frac{\ln (2)}{T_{1 / 2}}$ the decay constant and $\Delta t$ the correlation time window.

The total number of experimental correlations $N_{\exp }^{\mathrm{c}}$ as built in the first step can be expressed by the equation:

$N_{\mathrm{exp}}^{\mathrm{c}}=N^{\mathrm{tr}} \cdot\left(\frac{A_{\mathrm{true}}^{\mathrm{tr}}}{\varepsilon_{\mathrm{tr}}} I_{\mathrm{b}} \varepsilon_{\mathrm{c}}+A_{R 2}^{\mathrm{c}}+A_{\mathrm{bg}}^{c}\right) \Delta t+N_{\mathrm{true}}^{\mathrm{tr}} \cdot I_{\mathrm{b}} \varepsilon_{\mathrm{c}}\left(1-\mathrm{e}^{-\lambda \Delta t}\right)$,

where $N^{\mathrm{tr}}$ is the total number of triggers, $A_{\mathrm{true}}^{\mathrm{tr}}$ is the count rate of the true trigger events, $A_{R 2}^{\mathrm{c}}$ of randomly correlated 
events of the type " $R_{2}$ ", $A_{\mathrm{bg}}^{c}$ of background events and $\varepsilon_{\mathrm{tr}}$ the trigger detection efficiency. The first term at the righthand side of this equation represents exactly the total number of random correlations. ${ }^{2}$

The total number of experimentally deduced random correlations $N_{\text {exp }}^{r}$ as built in the second step can be expressed by the equation:

$$
\begin{aligned}
N_{\text {exp }}^{r}= & \frac{\sum_{i=1}^{N_{\text {cyc }}}\left[N^{\mathrm{tr}} \cdot\left(\frac{A_{\text {true }}^{\mathrm{tr}}}{\varepsilon_{\mathrm{tr}}} I_{\mathrm{b}} \varepsilon_{\mathrm{c}}+A_{R 2}^{\mathrm{c}}+A_{\mathrm{bg}}^{c}\right) \Delta t\right]_{i}}{N_{\mathrm{cyc}}} \\
& +\frac{N_{\mathrm{true}}^{\mathrm{tr}} \cdot I_{\mathrm{b}} \varepsilon_{\mathrm{c}}\left(1-\mathrm{e}^{-\lambda \Delta t}\right)}{N_{\mathrm{cyc}}},
\end{aligned}
$$

where $N_{\text {cyc }}$ is the total number of implantation-decay cycles.

The true correlations are experimentally estimated by subtracting the deduced random correlations $N_{\text {exp }}^{r}$ from the experimental correlated events $N_{\text {exp }}^{\mathrm{c}}$ :

$N_{\text {sub }}^{\mathrm{c}}=N_{\mathrm{exp}}^{\mathrm{c}}-N_{\exp }^{r}$

leading to

$N_{\text {sub }}^{\mathrm{c}}=N_{\text {true }}^{\mathrm{c}} \cdot\left[1-\frac{1}{N_{\mathrm{cyc}}}\right]$.

A systematic error term of $\frac{1}{N_{\text {cyc }}}$ appears. This means that as a first condition $N_{\text {cyc }} \gg 1$ has to be fulfilled.

The second condition is related to the count rates of trigger and correlated events. The experimentally estimated true correlations will only be an accurate estimate as long as its statistical uncertainty $\delta N_{\text {sub }}^{\mathrm{c}}$ is small enough with respect to the statistical uncertainty $\delta N_{\text {true }}^{\mathrm{c}}$ of the true correlations. This is formulated as

$\delta N_{\text {sub }}^{\mathrm{c}}<\alpha \cdot \delta N_{\text {true }}^{\mathrm{c}}$

with $\alpha$ a factor that is deduced from simulations based on the Monte-Carlo method. Using the first condition that $N_{\text {cyc }} \gg 1$, Eq. (2) and the assumption that the correlation time window $\Delta t$ is chosen large enough such that $\mathrm{e}^{-\lambda \Delta t}$ becomes negligibly small, this results in the second condition:

$A_{R 2}^{\mathrm{c}}+A_{\mathrm{bg}}^{c}<\left[\frac{\alpha^{2}-1}{\Delta t} \cdot \frac{N_{\mathrm{true}}^{\mathrm{tr}}}{N^{\mathrm{tr}}}-\frac{A_{\mathrm{truu}}^{\mathrm{tr}}}{\varepsilon_{\mathrm{tr}}}\right] \cdot I_{\mathrm{b}} \varepsilon_{\mathrm{c}}$.

From the simulations it is shown that $\alpha<\sqrt{5}$ is a safe limit in order that half-life fits are still reliable.

The first two terms at the left-hand side of the inequality are related to the purity of the beam and the background conditions for the correlated events, while the ratio $\frac{N_{\text {true }}^{\text {true }}}{N^{\text {tr }}}$ in the first term at the right-hand side of the inequality is related to the purity of the beam and the background conditions for the trigger events. The ratio $\frac{A_{\text {true }}^{\mathrm{tr}}}{\varepsilon_{\mathrm{tr}}}$ in the second term at the right-hand side of the inequality will make the correlation technique obsolete when the beam intensity

\footnotetext{
${ }^{2}$ More complete would be writing $\left(N_{\mathrm{true}}^{\mathrm{tr}}-1\right) / \Delta t_{\text {meas }}$ instead of $A_{\mathrm{true}}^{\mathrm{tr}}$, with $\Delta t_{\text {meas }}$ the total measuring time, in order to take into account the true correlation that is neglected here.
}

is too high. The correlation time window $\Delta t$ is chosen in function of the correlation half-life. A typical choice is $\Delta t=4 \cdot T_{1 / 2}$. Then, in perfect conditions, which means that $A_{R 2}^{\mathrm{c}}=A_{\mathrm{bg}}^{\mathrm{c}}=0 \mathrm{~Hz}$ and all trigger events are true trigger events $\left(N^{\text {tr }}=N_{\text {true }}^{\text {tr }}\right)$, Eq. (7) reduces to

$\frac{A_{\mathrm{true}}^{\mathrm{tr}}}{\varepsilon_{\mathrm{tr}}}<\frac{1}{T_{1 / 2}}$.

\section{Experimental results: a case study}

The correlation technique as described in Section 3 has been applied for the first time on LISOL data of the mass $A=67 \mathrm{Fe}-\mathrm{Co}-\mathrm{Ni}$ decay chain, where the lasers had been tuned subsequently on resonance to $\mathrm{Fe}, \mathrm{Co}$ and $\mathrm{Ni}$ and turned off (called lasers off). From comparing the lasers on Co with the lasers off data, prompt $\beta-\gamma$ coincidences confirmed the intense $694 \mathrm{keV} \gamma$-ray transition in ${ }^{67} \mathrm{Ni}$ (see Fig. 3) as was seen already in [11], in which the reported ${ }^{67} \mathrm{Co}$ half-life is $425(20) \mathrm{ms}$.

However, for both the $\mathrm{Fe}$ and Co data sets, an intense $492 \mathrm{keV} \gamma$-ray was observed in the single $\gamma$ spectrum, while it disappeared completely requiring coincidences with a $\beta$ particle. Neither were found gammas in coincidence with the $492 \mathrm{keV} \gamma$-events. As a result, at this stage, it was already clear that the $492 \mathrm{keV}$ transition is not situated in $\mathrm{Fe}$, but there was still no way to conclude whether the transition takes place in Co or Ni. Therefore, the described correlation technique has been applied.

Fig. 4 shows the three consecutive steps of building a random-free correlated histogram for single $\gamma$-events in a time window of $1 \mu$ s to $200 \mathrm{~ms}$ before $\beta-694 \mathrm{keV}$ trigger events. The spectra were built from the data taken with the lasers tuned resonantly on $\mathrm{Fe}$ and in a cycle of $10 \mathrm{~s}$ implantation, $0 \mathrm{~s}$ decay and a tape move after every cycle. The top spectrum shows all correlated events, the middle spectrum shows the approximated randomly correlated events and the bottom spectrum shows the random-free correlations. In the first step the spectrum shows a

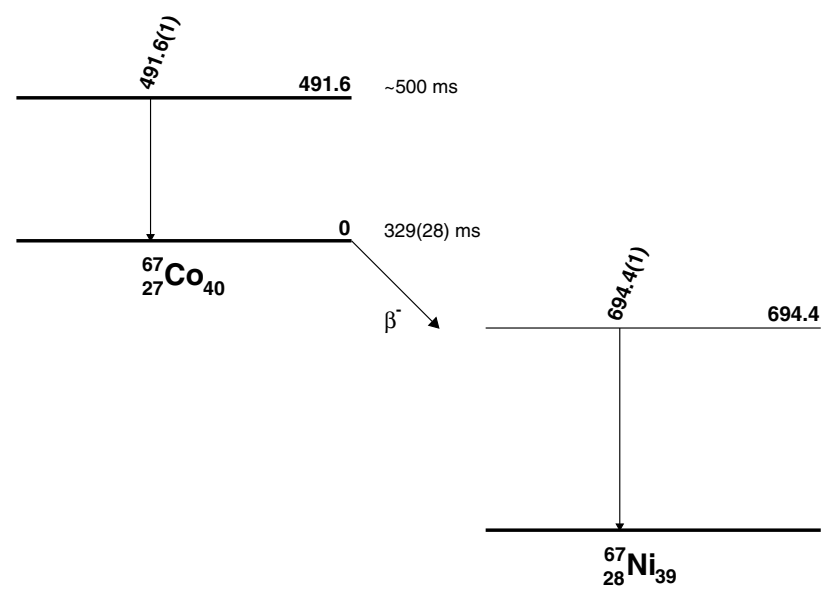

Fig. 3. Mass 67 decay chain resulting from the present study. 


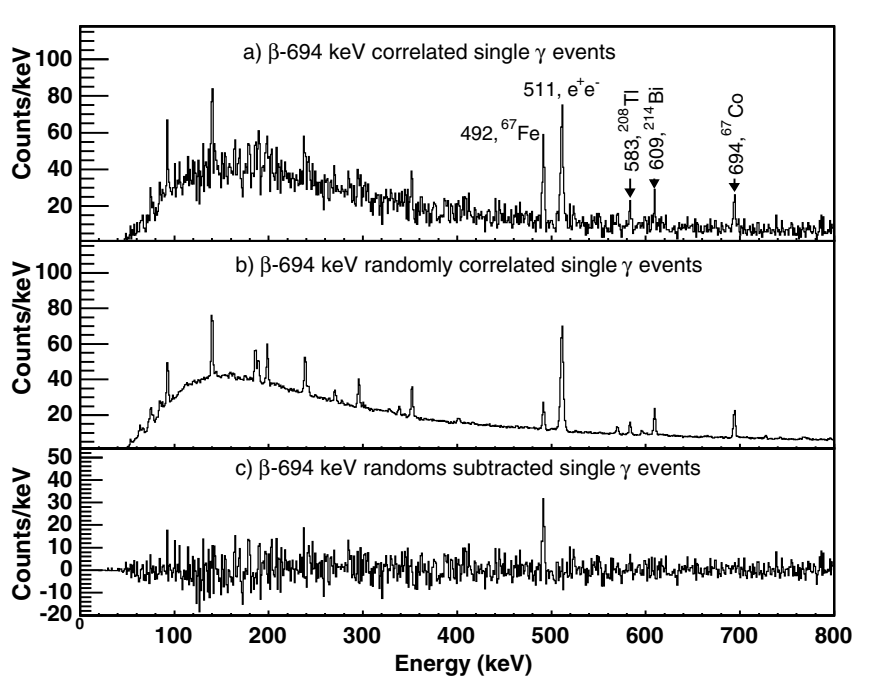

Fig. 4. Different steps of the correlation technique illustrated for the example of single $\gamma$-events in a time window of $1 \mu$ s to $200 \mathrm{~ms}$ before $\beta$ $694 \mathrm{keV}$ trigger events.

$492 \mathrm{keV}$ line, the uncorrelated $511 \mathrm{keV}$ line, other background lines and even the $694 \mathrm{keV}$ line itself. The presence of the last two lines is already evidence that the spectrum contains randomly correlated events and it is not clear if the observed $492 \mathrm{keV}$ line is physically correlated. In the second step the same lines appear again as the approximated randomly correlated events, but the ratio of the $492 \mathrm{keV}$ line intensity to the intensity of any other line is clearly different compared to the spectrum containing all correlations. Note also that the statistical fluctuations are much smaller in the second spectrum, because by definition the statistics is higher by a factor $N_{\text {cyc }}=19,409$, the total number of cycles in the Fe data. After subtraction of the middle spectrum from the top spectrum, only the $492 \mathrm{keV}$ line remains on a zero background level, shown in the bottom spectrum. This proves that the $492 \mathrm{keV}$ transition takes place before a $\beta-694 \mathrm{keV}$ event and thus in ${ }^{67} \mathrm{Co}$. Because the spectrum only shows a $492 \mathrm{keV}$ line, this proves also that the isomeric state decays internally through $\gamma$-emission to the ground state as shown in Fig. 3.

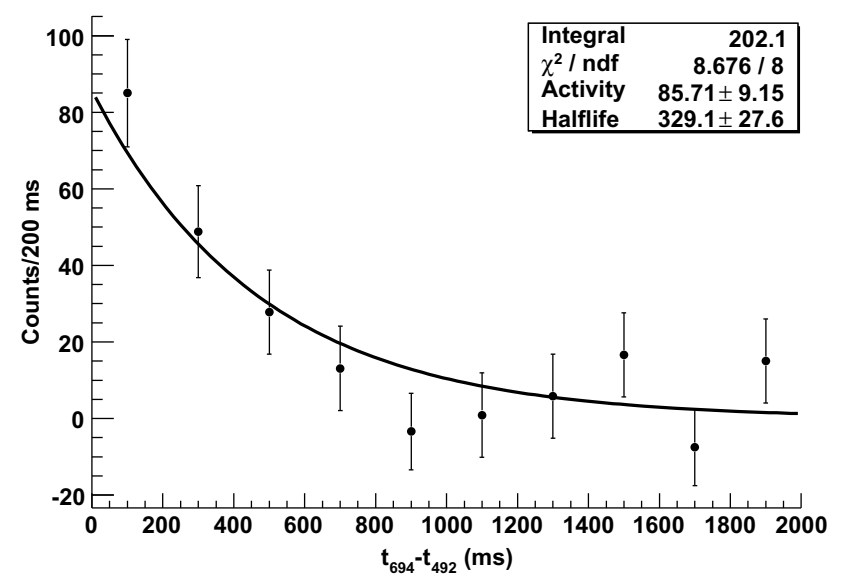

Fig. 5. Single exponential fit of the $492 \mathrm{keV}$ events coming before $\beta$ $694 \mathrm{keV}$ trigger events on a reversed time axis.
Apart from proving that the correlation exists, one can also deduce the half-life of the ground state of ${ }^{67} \mathrm{Co}$ by fitting the random-free correlated $492 \mathrm{keV}$ peak integrals as a function of the time difference $t_{694}-t_{492}$ between the correlated $492 \mathrm{keV}$ events and the trigger $\beta-694 \mathrm{keV}$ events, as shown in Fig. 5. Each data point represents the $492 \mathrm{keV}$ peak integral in the corresponding random-free correlation histogram, e.g., the $492 \mathrm{keV}$ integral from the spectrum in Fig. $4 \mathrm{c}$ is the first data point. The correlations satisfy the two conditions that were deduced in Section 4: $N_{\text {cyc }}=$ $19,409 \gg 1$ and working out numerically the left and right hand-side of inequality (7) for $\Delta t=2 \mathrm{~s}$ gives $0.08 \mathrm{~Hz}<$ $0.15 \mathrm{~Hz}$. Note that the integrals are statistically fluctuating around zero for the last six data points indicating a good control of the random correlations. In this way it was possible to determine the half-life of the ground state of ${ }^{67} \mathrm{Co}$ as $329(28) \mathrm{ms}$ with a simple single exponential relationship. The disagreement with the half-life of 425(20) ms [11] stems from the fact that in [11] the presence of the isomeric level was unknown.

A third application of the correlation technique is the determination of branching ratios.

\section{Conclusions}

A new segmented gamma setup consisting of two MINIBALL clusters and a new data-acquisition system, based on digital XIA-DGF4C electronics, in the $\beta \gamma$-detection setup at LISOL, has been discussed.

A new correlation technique has been developed and its limits are specified. In combination with weak, yet pure beams and low background conditions, the technique offers the possibility to correlate signals containing a large noncorrelated component like single betas or gammas, or $\beta$ coincident gammas up into the seconds range. The purity of the beam is realized by making use of resonant laser-ionization, in combination with mass separation. The correlations are able to deliver information on the placement of long-lived isomers in a decay chain, on the half-life of isomeric and ground states in a simple single exponential relationship and on branching ratios.

Experimental results, showing the strength of the technique, have been presented on the mass $A=67 \mathrm{Fe}-\mathrm{Co}-$ $\mathrm{Ni}$ decay. Using the correlations, a long-lived isomeric state at $492 \mathrm{keV}$ has been identified in ${ }^{67} \mathrm{Co}$, making an internal $\gamma$-ray transition to the ground state, which subsequently $\beta$ decays with a half-life of 329(28) ms.

This correlation technique in the seconds range could be used provided that the beams are weak and pure enough and the background level is low enough, as formulated by Eq. (7).

\section{Acknowledgements}

We acknowledge the support by the European Commission within the Sixth Framework Programme through 
I3-EURONS (contract no. RII3-CT-2004-506065), BriXIUAP P6/23, FWO-Vlaanderen (Belgium) , GOA/2004/ 03 and the Foundation for Polish Science (AK).

\section{References}

[1] A.N. Andreyev et al., Nucl. Instr. and Meth. A 533 (2004) 409.

[2] E.S. Paul et al., Phys. Rev. C 51 (1995) 78.

[3] O. Sorlin et al., Nucl. Phys. A 669 (2000) 351.
[4] D. Smirnov et al., Nucl. Instr. and Meth. A 547 (2005) 480.

[5] Yu. Kudryavtsev, M. Facina, M. Huyse, J. Gentens, P. Van den Bergh, Nucl. Instr. and Meth. B 204 (2003) 336.

[6] M. Facina et al., Nucl. Instr. and Meth. B 226 (2004) 401.

[7] L. Weissman et al., Nucl. Instr. and Meth. A 423 (1999) 328.

[8] J. Eberth et al., Prog. Part. Nucl. Phys. 46 (2001) 389.

[9] $<$ http://www.xia.com/DFG-4C.html $>$.

[10] $<$ http://www.wavemetrics.com/products/igorpro/igorpro.htm>.

[11] L. Weissman et al., Phys. Rev. C 59 (1999) 2004. 